\title{
Pedestal Structure and Energy Confinement Studies on TCV
}

\author{
U. A. Sheikh ${ }^{1}$, M. Dunne ${ }^{2}$, L. Frassinetti ${ }^{3}$, P. Blanchard ${ }^{1}$, \\ B. P. Duval ${ }^{1}$, B. Labit ${ }^{1}$, A. Merle ${ }^{1}$, O. Sauter ${ }^{1}$, C. Theiler ${ }^{1}$, \\ C. Tsui ${ }^{1}$, the TCV Team ${ }^{4}$ and the EUROfusion MST1 Team ${ }^{5}$ \\ ${ }^{1}$ École Polytechnique Fédérale de Lausanne (EPFL), Swiss Plasma Center \\ (SPC), CH-1015 Lausanne, Switzerland \\ ${ }_{2}^{2}$ Max Planck Institute für Plasmaphysik, Garching, Germany \\ ${ }^{3}$ KTH Royal Institute of Technology, Stockholm, Sweden \\ ${ }^{4}$ See author list of S. Coda et al 2017 Nucl. Fusion 57102011 \\ ${ }^{5}$ See author list of H. Meyer et al 2017 Nucl. Fusion 57102014 \\ E-mail: umar.sheikh@epfl.ch \\ July 2018
}

Abstract. High external gas injection rates are foreseen for future devices to reduce divertor heat loads and this can influence pedestal stability. Fusion yield has been estimated to vary as strongly as $T_{e, p e d}^{2}$ so an understanding of the underlying pedestal physics in the presence of additional fuelling and seeding is required. To address this, a database scanning plasma triangularity, fuelling and nitrogen seeding rates in neutral beam (NBH) heated ELM-y H-mode plasmas was constructed on TCV. Low nitrogen seeding was observed to increase pedestal top pressure but all other gas injection rates led to a decrease. Lower triangularity discharges were found to be less sensitive to variations in gas injection rates. No clear trend was measured between plasma top $\mathrm{P}_{e}$ and stored energy which is attributed to the non-stiffness of core plasma pressure profiles.

Peeling ballooning stability analysis put these discharges close to the ideal MHD stability boundary. A constant for $D$ in the relation pedestal width $w=D \sqrt{\beta_{\theta}^{P e d}}$, was not found. Experimentally inferred values of $D$ were used in EPED1 simulations and gave good agreement for pedestal width. Pedestal height agreed well for high triangularity but was overestimated for low triangularity. IPED simulations showed that relative shifts in pedestal position were contributing significantly to the pedestal height and were able to reproduce the measured profiles more accurately. 


\section{Introduction}

It is currently foreseen that next step fusion devices will operate in the high confinement plasma mode (Hmode) using external gas injection to reduce divertor heat fluxes to acceptable levels. Puffing of impurities and additional fuelling can have a significant influence on the edge transport barrier, altering the region of strong temperature and density gradients termed the pedestal $[1,2,3]$. The height of the pedestal is an essential parameter in assessing the performance of a machine and extrapolating towards future devices. Modelling has shown that fusion yield could scale as strongly as $T_{p e d}^{2}[4]$ making the pedestal an important topic for fusion reactor research.

Fuelling and impurity seeding has been seen to influence pedestal and global performance in several machines. ASDEX Upgrade (AUG) and JET experiments showed that increased fuelling can reduce pedestal performance. AUG explains this through a change in the high field side high density (HFSHD) $[5,6]$ region, leading to an outward shift of the pedestal position and a consequent degradation in pedestal stability[7]. There is no definitive explanation for pedestal degradation on JET but a clear correlation was observed for a relative shift between the temperature and density pedestal positions $[8,9]$.

Impurity seeding has led to increases in pedestal performance in metal walled machines. Nitrogen seeding on JET-ILW resulted in improved pedestal height to near JET-C levels [10, 11]. AUG achieved similar results with nitrogen seeding [7]. Furthermore, neon and carbon seeding experiments on AUG both resulted in improved pedestal performance [12, 13]. Increases in pedestal height observed in all impurity seeding experiments on AUG have been linked to a reduction of the HFSHD region[7]. JET is continuing investigations to try to link the HFSHD region to pedestal performance with impurity seeding.

TCV has an open geometry with carbon walls compared to AUG and JET, which have closed divertors and metal walls. A HFSHD region is not expected[14]. Furthermore, TCV has unique shaping capabilities that allow a wider range of plasma shapes to be explored. The aim of this study is to use TCV to explore the effects of combinations of impurity seeding, fuelling and plasma shape. For this, two type-IELMy H-mode scenarios with a different triangularity were esbtalished using the newly commissioned neutral beam heating (NBH) system[15]. A range of deuterium fuelling and nitrogen seeding scenarios were explored and their influence upon pedestal characteristics were modelled in terms of transport and MHD.

The EPED1 model for pedestal stability predicts pedestal top pressure through the kinetic ballooning mode (KBM), and the peeling ballooning (PB) limits[16]. A canonical pedestal cycle begins with a steepening of the pressure pedestal until a maximum pedestal gradient is reached. This limit is dictated by transport. In EPED1, this limit is modelled using KBM stability as a proxy[16]. Once this maximum gradient is reached, the plasma remains on the KBM limit but the pedestal height can still increase through a widening of the pedestal width, $w$. The KBM constraint suggests that the pedestal width increases as $w=D \sqrt{\beta_{\theta}^{P e d}}$, as experimentally observed in many devices[17]. This approximation does not account for other factors such as micro-turbulence but has been supported by experimental measurements on DIII-D, AUG and Alcator C-mod (C-MOD). The parameter $D$ is therefore related also to the pedestal gradient (for the same pedestal height, a larger $D$ gives a lower gradient) and hence, at least in part, to the transport. In EPED1, the value of $D$ is set to 0.076 , as inferred from low collisionality DIII-D data with ITER like plasma parameters[16]. Other values for $D$ were obtained on other devices; 0.11 for AUG and 0.84 for C-MOD[16]. The scalar $D$ was found to vary on JET with values ranging from 0.05 to $0.2[8]$. This implies that using a fixed value of $D$ in the product $D \sqrt{\beta_{\theta}^{\text {Ped }}}$ was insufficient in capturing transport effects.

With the gradient set through the KBM, in this model the pedestal width continues to grow until the PB limit (set by ideal MHD) is reach. Growth of the pedestal width at a constant gradient leads to an increasing pedestal height. The maximum pedestal pressure is thus defined by the intersection of the KBM and PB limits. Beyond this limit the pedestal collapses through a type I edge localised mode (ELM) and the pedestal cycle begins again. Figure 1 presents a schematic of the KBM and PB limits as a function of pedestal width and pedestal top pressure. Variation of the fit parameter $D$ changes the gradient of the profiles and therefore mimics what would happen if transport were to change. This is shown by the dashed lines in Figure 1. The ideal MHD limit, ergo the PB limit, is modified by several parameters such as plasma shape, pedestal position and normalised $\beta$ and is shown by the solid lines in Figure 1. It should be noted that EPED does not account for the pedestal position and an extension of the program, IPED[19], is required to study the influence of position on the $\mathrm{PB}$ limit. Employing a fixed value of $D$, i.e. fixing the transport coefficient in the EPED1 model allows for the influence of global parameters on the ideal MHD limit to be studied. Conversely, scanning $D$ allows for the effect of transport on the profiles to be observed and it was for these reasons EPED1 was selected as the model for this study. 


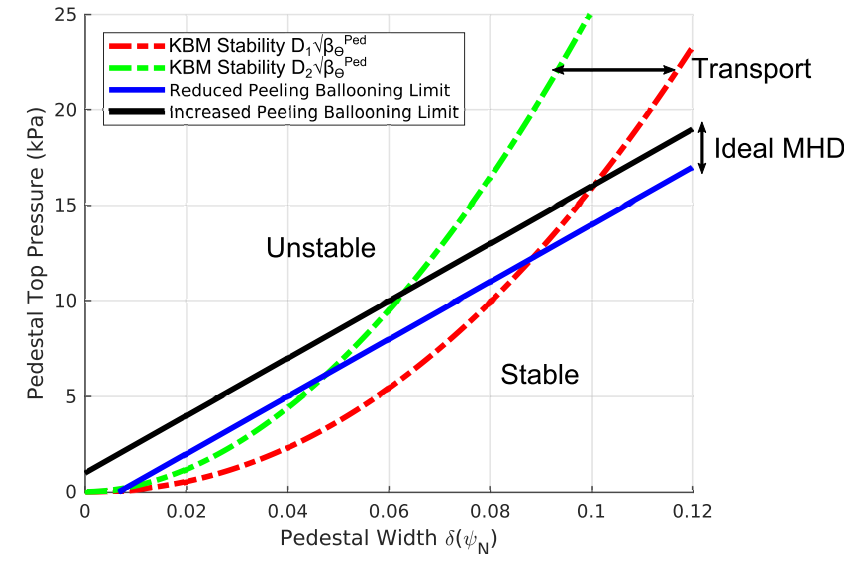

Figure 1. Schematic of peeling ballooning and kinetic ballooning mode stability.

\section{Experiment Description}

This TCV study used two plasma lower single null configurations with upper triangularities of 0.2 and 0.55 and a lower triangularity of 0.59 . Nominal 1.0 MW of NBH power was applied between 0.8 and $1.3 \mathrm{~s}$ achieving stationary ELM-y H-mode. Figure 2 presents an overview of the discharge parameters. The H-mode phase is marked by an increase in line averaged density and the presence of the ELMs inferred from D-alpha emission. The fuelling valve was shut throughout the H-mode phase of the discharge and wall recycling remained constant as evidenced by the steady plasma density. Helium glow discharges were carried out between experiments ensuring repeatable wall conditions. In a repeat discharge, the ELMs were determined to be of type I as the frequency decreased from over $110 \mathrm{~Hz}$ to approximately $60 \mathrm{~Hz}$ as $\mathrm{NBH}$ power was decreased from 1.0 MW to $500 \mathrm{~kW}$.

A sequences of discharges with a range of deuterium fuelling rates, nitrogen seeding rates with no fuelling, and nitrogen seeding rates with a 4.4 mbar $\mathrm{L} / \mathrm{s}$ fuelling during $\mathrm{H}$-mode were performed for both triangularities. The flow rates utilized are presented in Table 1. Reference discharges for each triangularity were conducted with both fuelling and nitrogen seeding valves remaining completely closed during the H-mode phase of the discharge. A total of six scans were performed exploring three sets of fuelling and seeding rates for the two plasma triangularities.

Table 1. Nominal fuelling and seeding rates utilized.

\begin{tabular}{lllllll}
\hline $\mathrm{D}_{2}$ Rates $($ mbarL $/ s)$ & 0.0 & 1.1 & 4.4 & 15.7 & 31.2 & \\
\hline $\mathrm{N}_{2}$ Rates $($ mbarL $/ s)$ & 0.0 & 0.5 & 2.3 & 5.0 & 8.2 & 11.6 \\
\hline
\end{tabular}

TCV's high resolution Thomson Scattering system was used to measure $\mathrm{T}_{e}$ and $\mathrm{n}_{e}$ at the locations
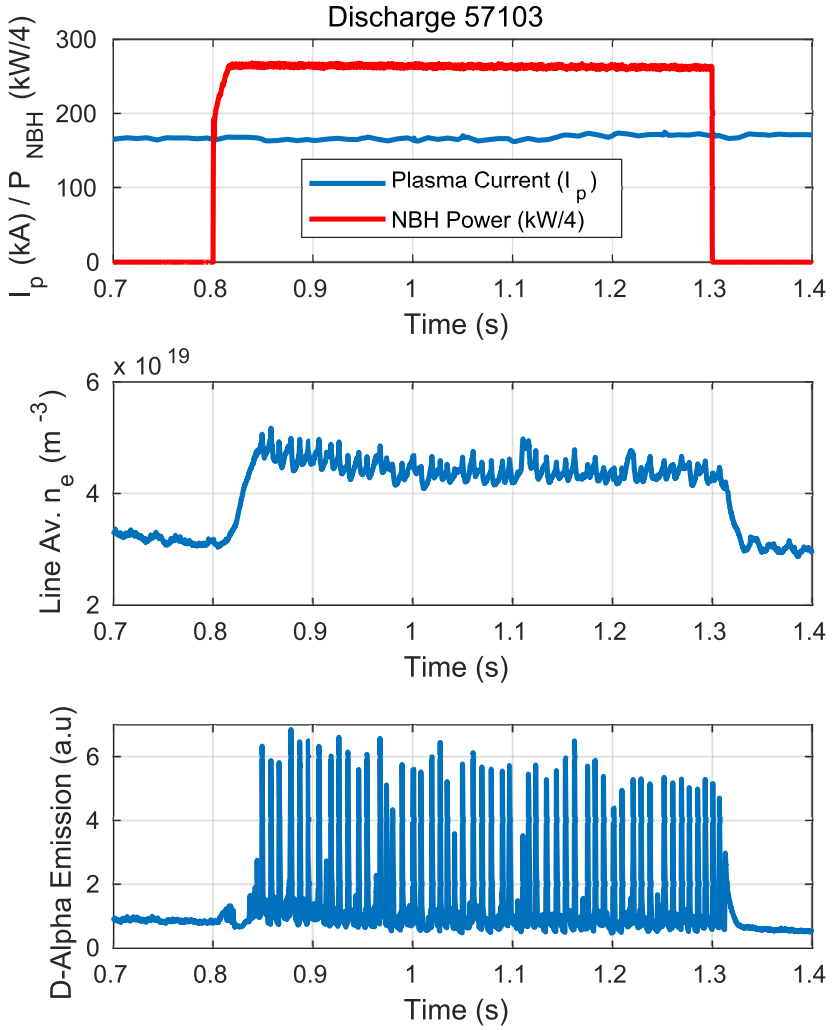

Figure 2. Reference discharge overview with plasma current, nominal beam heating power, line averaged density and D-alpha emission.

indicated on the low triangularity reference equilibrium presented in Figure 3. The TCV Thomson Scattering system features enhanced spatial resolution with the low $\mathrm{T}_{e}(10 \mathrm{eV})$ measurement capability near the seperatrix allowing for enhanced measurements of the pedestal. The temporal resolution of the system is the pulse rate of three lasers, which are each able to fire once every $50 \mathrm{~ms}$. All measurements obtained within the last $30 \%$ of the ELM cycle were combined and fitted with an mtanh function as prescribed in [20] with the core profiles fitted by a 4 th order polynomial. Profiles were radially displaced to obtain a seperatrix temperature of $50 \mathrm{eV}$. This value was obtained from a previous TCV database scaling with $1.0 \mathrm{MW}$ of power crossing the seperatrix [21, 22]. This approach was also used on other machines to minimise any systematic errors caused by equilibrium reconstructions. The seperatrix temperatures used on AUG and JET was $100 \mathrm{eV}$ and $50 \mathrm{eV}$ on MAST-U. A sample fit of the $\mathrm{T}_{e}$ pedestal for the low triangularity reference discharge is presented in Figure 3.

\section{Results}

The results section is divided into two parts; effects of seeding and fuelling on pedestal height and 

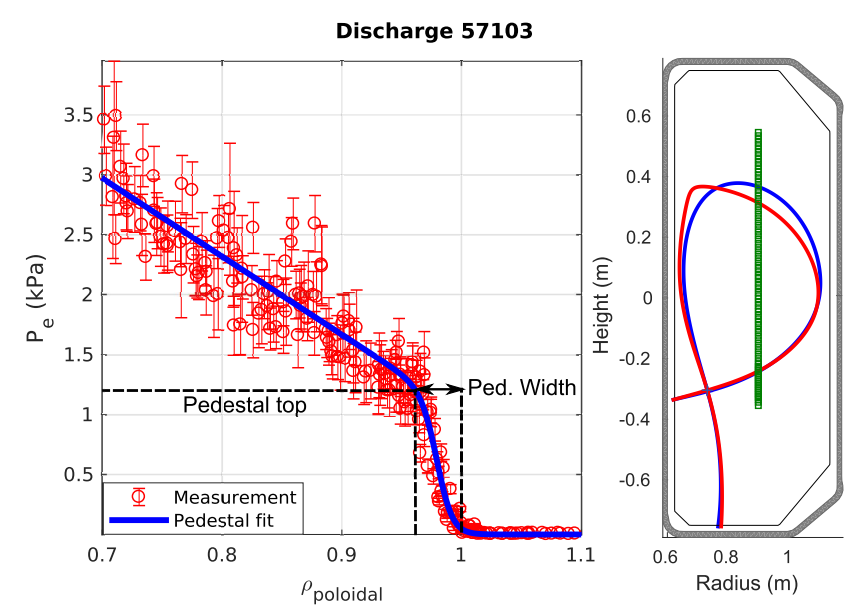

Figure 3. Right - Reference high (red) and low (blue) triangularity plasma equilibria superimposed with Thomson Scattering measurement locations in green squares. Left $-\mathrm{P}_{e}$ measurements and the fitted profile.

the correlation between pedestal height and plasma performance. The discharges are presented as three sets for each plasma triangularity; fuelling scan, seeding scan and seeding scan with constant fuelling. For simplicity, only the complete individual profiles from the fuelling scan at high triangularity are presented and for the remainder of the analysis the pedestal top values from the mtanh fits are used.

Mean normalised $\beta$ values of 1.6 and 1.8 were measured for the low and high triangularity discharges respectively. These values did not deviate by more than $15 \%$ throughout the seeding and fuelling scans, ensuring a valid comparison between the discharges. Pedestal collisionality was observed to range from 1 to 5 and this is in good agreement with other verified experiments that have presented values between 0.8 and $5[2,18,23]$.

\subsection{Effects of Fuelling and Seeding on the Pedestal}

Pedestal profiles of $T_{e}, n_{e}$ and $P_{e}$ are presented in Figure 4 for discharges conducted using the high triangularity plasma shape at a series of the fuelling rates. The pedestal density increased with fuelling rate and was accompanied by an outward shift of pedestal position. No shift in density pedestal position without a significant change in other pedestal parameters was observed. So a direct study of the impact of the pedestal position on pedestal height, as in AUG[7] and JET[9], was not possible. The reduction in pedestal height seen with an outward shift is qualitatively consistent with measurements in AUG and JET.

A reduction of over $50 \%$ in pedestal top $\mathrm{T}_{e}$ was measured across the gas scan and this was accompanied by an outward shift of the pedestal. Over this range, the pedestal top $\mathrm{n}_{e}$ increased by $30 \%$ with a
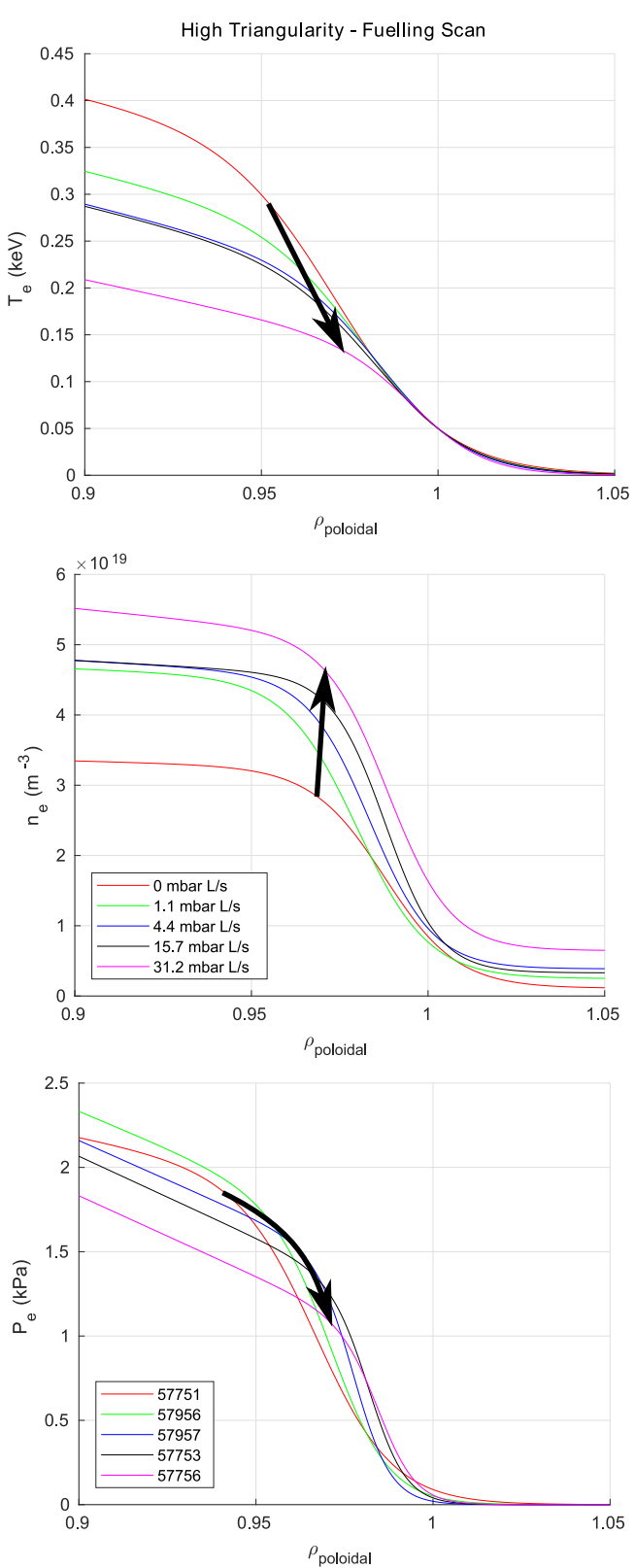

Figure 4. $T_{e}, n_{e}$ and $P_{e}$ pedestal profiles for the high triangularity fuelling scan. Black arrows indicate the direction of increasing fuelling.

weaker outward shift. The resulting pressure pedestal shifted outwards decreasing by approximately $50 \%$ with increased fuelling. This decrease is larger than the product of the $\mathrm{T}_{e}$ and $\mathrm{n}_{e}$ values and this is due to their relative radial shifts. Figure 5 presents the nominal pedestal top values from the profile fits and these results highlight the significant reduction in pedestal height with fuelling at high triangularity on TCV.

Figure 6 shows the pedestal top $\mathrm{P}_{e}$ values of each fuelling, nitrogen seeding and nitrogen seeding with constant fuelling discharge. As previously observed on JET, higher pedestals pressures were observed with 


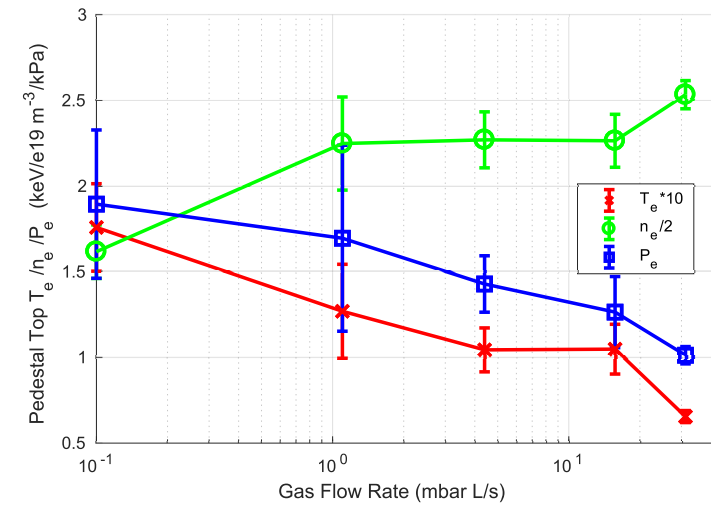

Figure 5. Pedestal top $T_{e}, n_{e}$ and $P_{e}$ values for varying fuelling rates at the high triangularity shape.

higher triangularity[8]. On TCV, peak pedestal top $\mathrm{P}_{e}$ was obtained at higher triangularity with a nitrogen seeding rate of $0.5 \mathrm{mbar} \mathrm{L} / \mathrm{s}$. Surprisingly, the lowest pedestal top $\mathrm{P}_{e}(80 \%$ lower $)$ was also observed with higher triangularity for the highest nitrogen seeding rate and constant fuelling. It should be noted that two of the nitrogen seeding discharges contained an MHD mode and these have been removed from further analysis. In general, a relatively weaker influence of fuelling and seeding was observed on low triangularity discharges with a pedestal top $\mathrm{P}_{e}$ variation of $40 \%$. As observed on JET[11], high triangularity discharges on TCV are more sensitive to fuelling and seeding. Divertor heat load analysis for this dataset is ongoing but has no direct effect on the conclusions of the paper and is therefore omitted here.

\subsection{Correlation Between Pedestal Height and Plasma Performance}

The link between pedestal height and plasma performance was studied through a comparison of pedestal top $\mathrm{P}_{e}$ and total plasma stored energy obtained by the diamagnetic loop array on TCV. Figure 7 presents the experimental measurements of both parameters. A $40 \%$ variation in pedestal height and a $25 \%$ change in total stored energy was measured for low triangularity discharges with no clear trend between the two. The high triangularity discharges show a weak trend at lower pedestal top $\mathrm{P}_{e}$ and no clear trend above $1.2 \mathrm{kPa}$. The reference discharge produced the lowest stored energy whilst having the highest pedestal top pressure. This may result from low density lowering collisionality, leading to changes in $\mathrm{NBH}$ coupling and energy exchange between ions and electrons. An investigation of this is being undertaken with core transport and beam interaction modelling and is outside the scope of this study.

The absence of a clear trend between pedestal top $\mathrm{P}_{e}$ and plasma stored energy for both plasma shapes
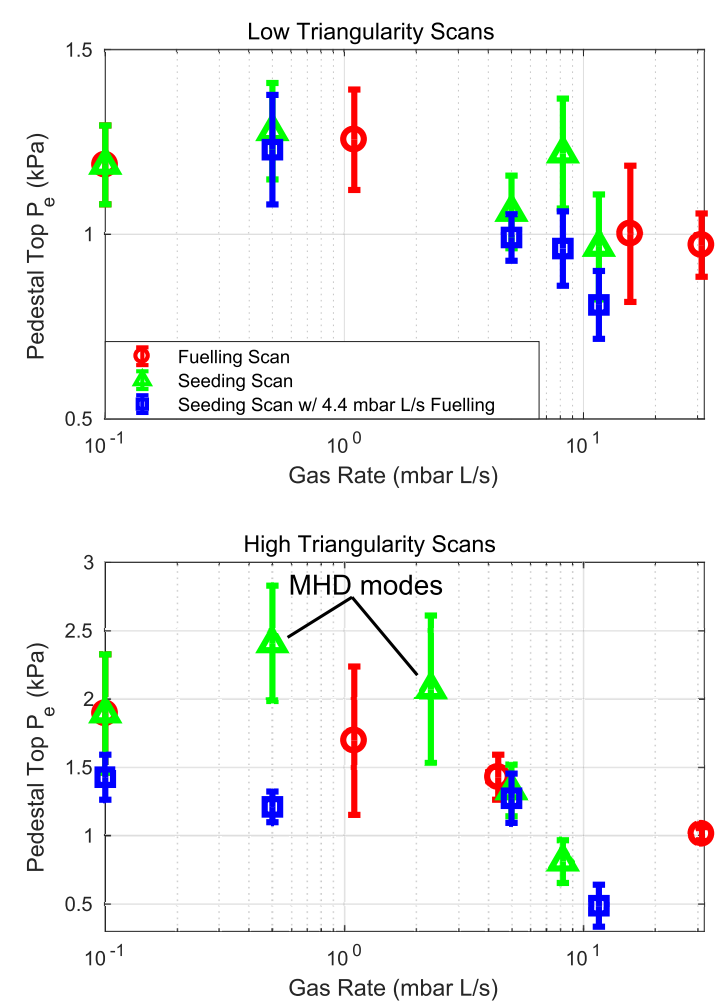

Figure 6. Pedestal top $\mathrm{P}_{e}$ for fuelling, seeding and seeding with constant fuelling scans at low (top) and high (bottom) triangularity plasma shapes. Discharges omitted from further analysis due to MHD modes are marked.

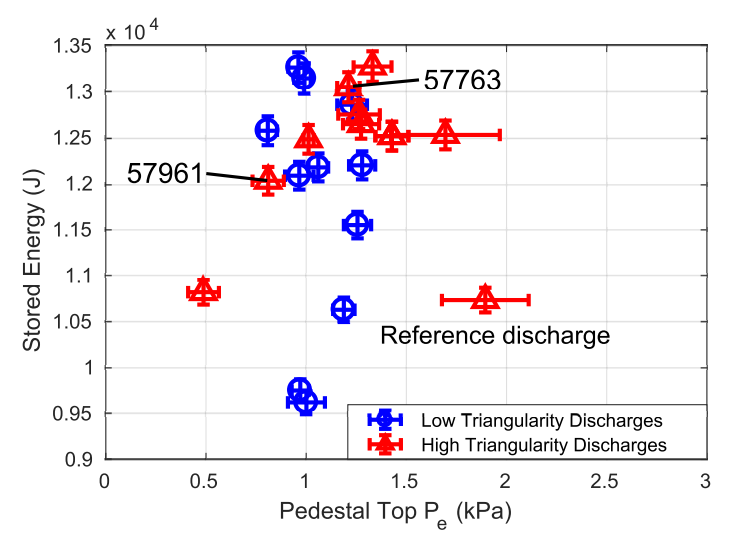

Figure 7. Pedestal top $\mathrm{P}_{e}$ and total stored plasma energy for all low triangularity discharges (blue circles) and high triangularity discharges (red triangles).

is attributed to changes in the bulk plasma pressure profiles, i.e. the core profiles are not very stiff. This is not necessarily due to the pedestal changing the core profile but may be influenced by seeding and fuelling gases altering plasma parameters such as collisionality and inductance. Figure 8 shows an example of this with two high triangularity discharges at high nitrogen seeding rates and a stored energy variation of less than $10 \%$ with pedestal top $\mathrm{P}_{e}$ variation of $40 \%$. The 
discharges presented are \#57763 (red) and \#57961 (green) with nitrogen seeding rates of $5.0 \mathrm{mbar} \mathrm{L} / \mathrm{s}$ and $8.2 \mathrm{mbar} \mathrm{L} / \mathrm{s}$ respectively. Both profiles have been normalised by their pedestal top $\mathrm{P}_{e}$ and discharge \#57961 shows significantly stronger pressure peaking in the core.

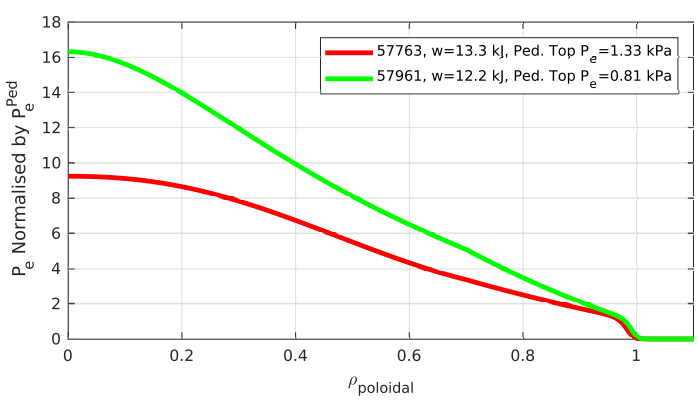

Figure 8. $\mathrm{P}_{e}$ profiles normalised by pedestal top $\mathrm{P}_{e}$ for two high triangularity discharges with similar stored energy but different pedestal heights. Discharge 57763 (red) and discharge 57961 (green).

\section{Comparison With the EPED1 model}

The primary assumption of the EPED1 model is that the pedestal is limited by ideal MHD. To validate this assumption a PB stability limit analysis was carried out using experimental measurements and EPED$\mathrm{CH}[24]$. High resolution equilibria were generated using the CHEASE code[25] and the pressure profiles were constrained by the fitted profiles from the Thomson Scattering system with an assumption of equal ion and electron temperatures. The parallel current density profiles were constrained by the bootstrap current calculated using the Sauter model[26]. Equilibria were then recalculated using CAXE[27] to generate the maximum current density $\left(J_{\|}\right)$for a given normalised pressure gradient $(\alpha)$. The PB stability was calculated for each equilibrium using KINX[28] for a range of toroidal mode numbers ranging from 5 to 80 and the stability boundary for each toroidal mode number was assumed to be at the ideal MHD limit, i.e. a growth rate of zero. Figure 9 presents the stability diagrams for three discharges from the dataset: the low and high triangularity reference discharges and a high triangularity discharge with high fuelling. These results show that the pedestal values achieved for both shapes are close to or on the $\mathrm{PB}$ stability limit, even in the presence of high fuelling. This result enables the use of EPED1 as tool to investigate the sensitivity of various plasma parameters on the performance of the pedestal on TCV.

EPED1 model assumes that the pressure pedestal width will increase as a function of $D \sqrt{\beta_{\theta}^{P e d}}$. To
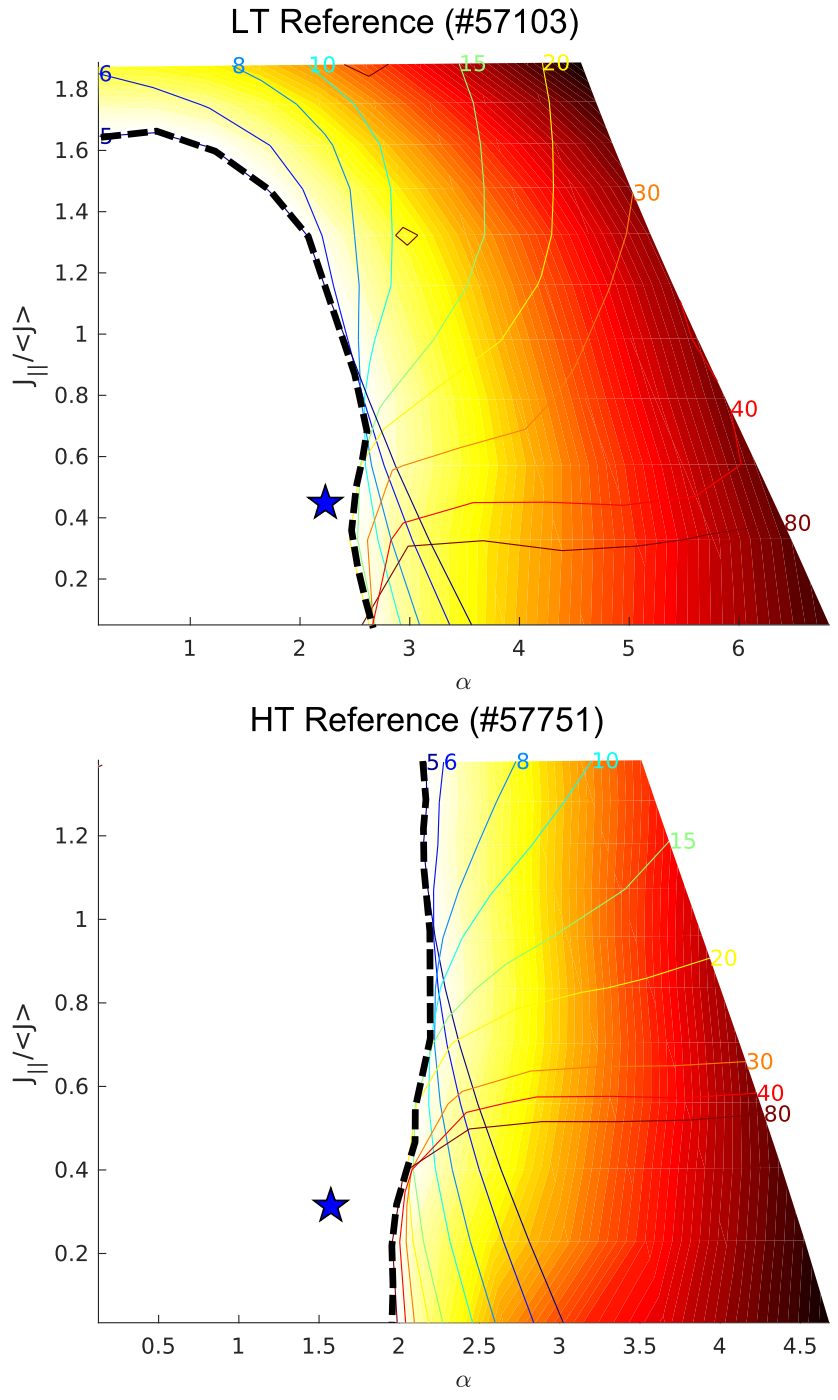

HT With Fuelling (\#57753)

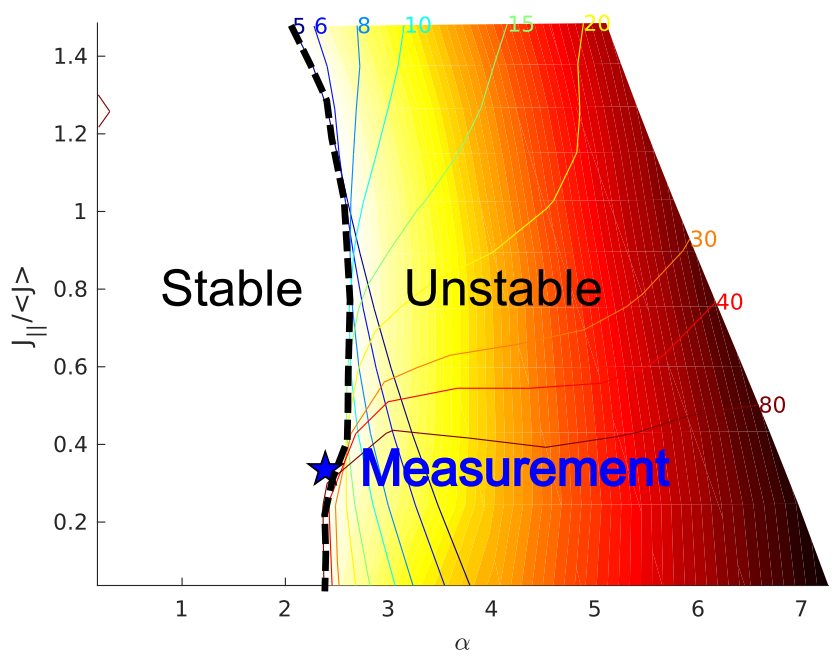

Figure 9. PB stability limit for the low triangularity (LT) reference discharge (top), high triangularity (HT) reference discharge (middle) and a high triangularity discharge with high fuelling (bottom). The stars indicate the measured value for each discharge and the dashed lines indicate the stability boundary. 


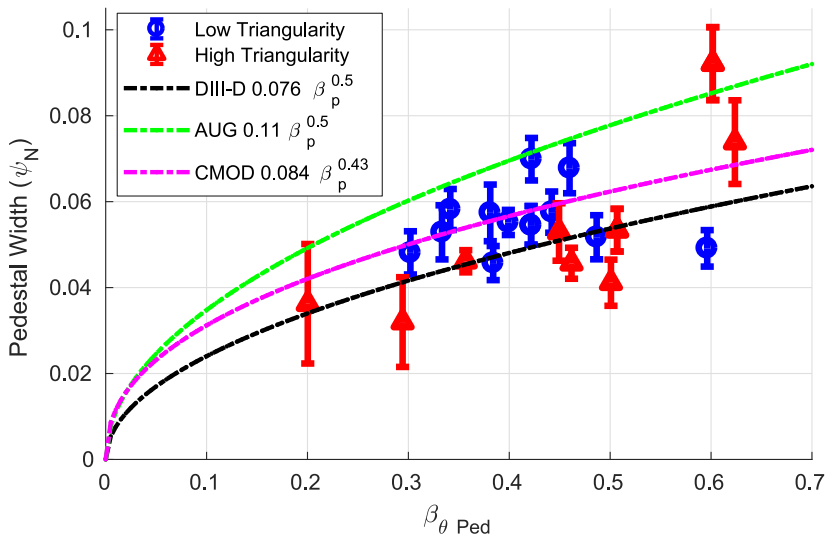

Figure 10. Pedestal width as a function of $\beta_{\theta}^{P e d}$. Low triangulartiy discharges shown in blue circles high triangularity discharges shown in red triangles. Comparisons with fits from DIII-D, AUG and CMOD superimposed with dashed lines.

validate this assumption, $\beta_{\theta}^{\text {Ped }}$ and pedestal width are presented in Figure 10 for all discharges. The fit constant from DIII-D (0.076) used in the EPED1 model is overlaid in black with values obtained from C-MOD and AUG also indicated. Although low triangularity discharges agree well for the range of fit constants, the high triangularity discharges show a larger spread and do not appear to follow the functional form. This implies that transport properties may be significantly changing with fuelling and seeding for high triangularity[17]. It is interesting to note that the large range in $D$ was also observed at JET[18].

EPED1 simulations using a constant $D$ value of 0.076 were produced and are presented in Figure 12. Using a constant value of $D$ resulted in small changes in pedestal top $\mathrm{P}_{e}$ and width across the dataset with differences of over a factor of two in comparison with measurements. Modelled scans of $\beta_{N}$, pedestal top $\mathrm{n}_{e}$ and $Z_{\text {eff }}$ also showed a weak influence of these parameters on pedestal top $\mathrm{P}_{e}$ $(<15 \%)$. Maintaining EPED input parameters at average values from the high triangularity scan, $D$ was scanned from 0.06 to 0.12 and this resulted in a pedestal top $\mathrm{P}_{e}$ change of approximately $65 \%$. This indicates that the parametrisation of transport through $D \sqrt{\beta_{\theta}^{P e d}}$ is highly sensitive to the fit parameter $D$. As $D$ is not observed to be a constant value, or have any evident trend across the dataset as shown in Figure 11, it was not possible to use EPED in a predictive capacity. Instead, values of $D$ were inferred for each TCV discharge using the measured pedestal width and retaining the $\sqrt{\beta_{\theta}^{\text {Ped }}}$ dependence that has been seen on DIII-D, AUG and C-MOD, albeit with different multipliers as previously mentioned. This allows testing of the EPED assumption $w \alpha \sqrt{\beta_{\theta}^{P e d}}$ without invoking complex transport modelling to ascertain D.

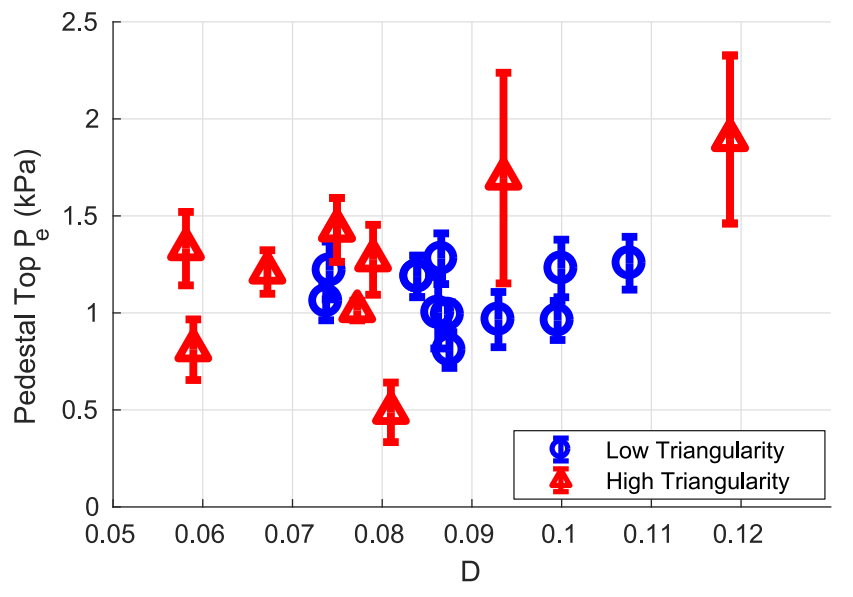

Figure 11. Pedestal top $\mathrm{P}_{e}$ as a function of the $\beta_{\theta}^{P e d}$ fit parameter $D$ calculated for each discharge. Low triangulartiy discharges (blue circles), high triangularity discharges (red triangles).

EPED simulations employing the experimentally inferred values of $D$ were then performed. Figure 12 top shows the computed and measured pedestal widths, which are in good agreement across the dataset. With the ideal MHD assumptions used in the EPED calculation, this result strongly suggests that these scenarios are on the PB stability limit. Furthermore, this result highlights the need for accurate accounting of transport to obtain the constant $D$ and compute an accurate pedestal width with EPED.

Figure 12 bottom shows a comparison of the computed and measured values of pedestal top $\mathrm{P}_{e}$. For the low triangularity discharges, the predicted values of pedestal top $\mathrm{P}_{e}$ overestimate the bulk of the measured values. Reasonable agreement is observed with the high triangularity discharges with the exception of one outlier. Possible explanations for this discrepancy may be that relative changes in pedestal position are not accounted for by the EPED model and/or the fixed scaling exponential of 0.5 for $\beta_{\theta}^{P e d}$.

The outlier (\#57960) and discharge \#57956, Figure 13, were at two extremes of the gas scans; discharge \#57956 (red) has low deuterium fuelling whilst discharge \#57960 (green) has high nitrogen seeding with constant deuterium fuelling. Discharge \#57956 has the same position for both the $\mathrm{n}_{e}$ and $\mathrm{T}_{e}$ pedestals, however, the $\mathrm{n}_{e}$ pedestal in discharge \#57960 has shifted outwards relative to the $\mathrm{T}_{e}$ pedestal. The $\mathrm{T}_{e}, \mathrm{n}_{e}$ and $\mathrm{P}_{e}$ profiles for both discharges are presented in Figure 13. The experimental measurements of pedestal top $\mathrm{P}_{e}$ vary by a factor of 3.5 between the two discharges. As EPED is not capable of resolving pedestal position, IPED was used to compute the effect of the relative shift in the $n_{e}$ and $T_{e}$ pedestal positions for discharge \#57960. 

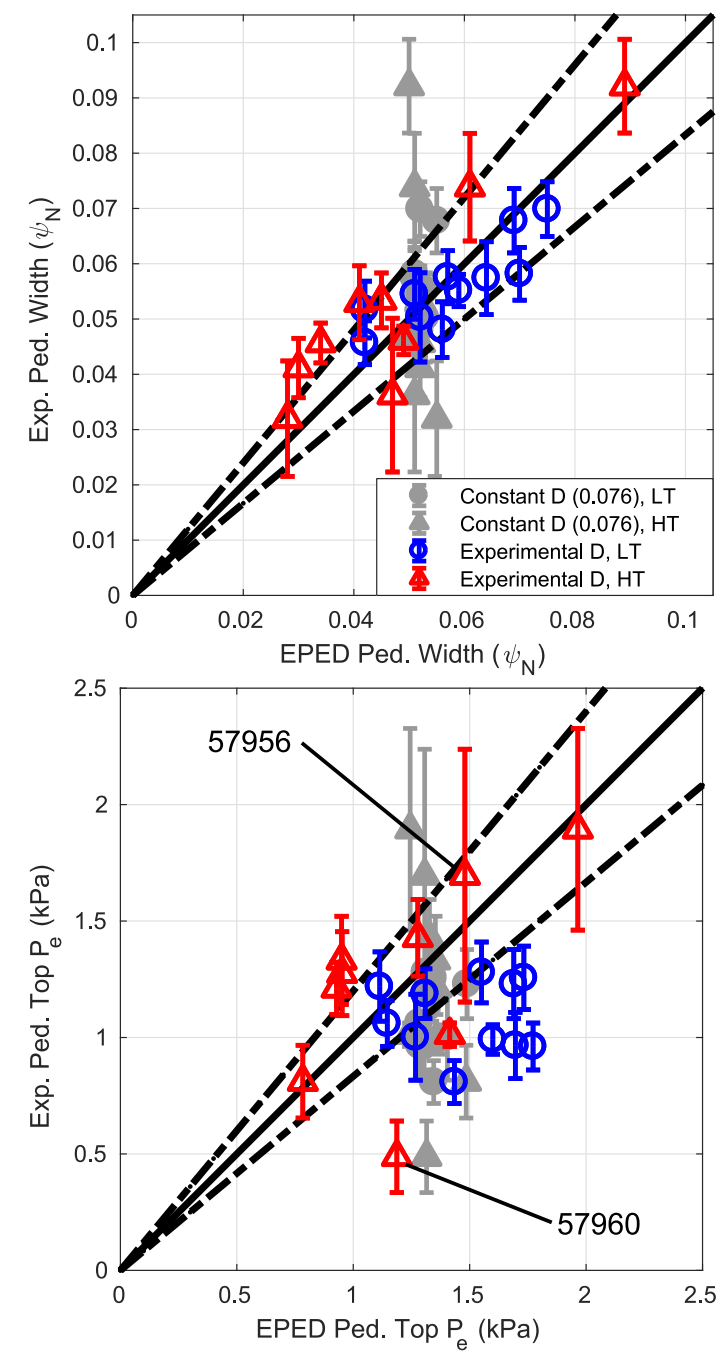

Figure 12. Pedestal width (top) and pedestal top $\mathrm{P}_{e}$ predicted by EPED1 (grey symbols) and EPED with experimentally measured values of $D$ (coloured symbols) in comparison with measured values. The solid lines indicate parity between measurements and simulations and the dashed lines indicate a $20 \%$ error margin. Low triangulartiy discharges (blue and grey circles), high triangularity discharges (red and grey triangles).

IPED was not required for discharge \#57956 as there is no relative shift between the $\mathrm{n}_{e}$ and $\mathrm{T}_{e}$ pedestals and therefore it would provide the same result as EPED.

Figure 13 bottom shows the IPED and EPED results with experimentally inferred values of $D$, superimposed on the measured $\mathrm{P}_{e}$ profiles. As expected, EPED significantly over estimates the pedestal top pressure for discharge \#57960. Including a $3 \%$ shift in relative position of the $\mathrm{n}_{e}$ profile resulted in a reduction of the predicted pedestal top $\mathrm{P}_{e}$ by almost $50 \%$, lowering the predicted pedestal pressure profile towards the measured values. This result highlights the need to accurately model pedestal position and account for this in pedestal height predictions.
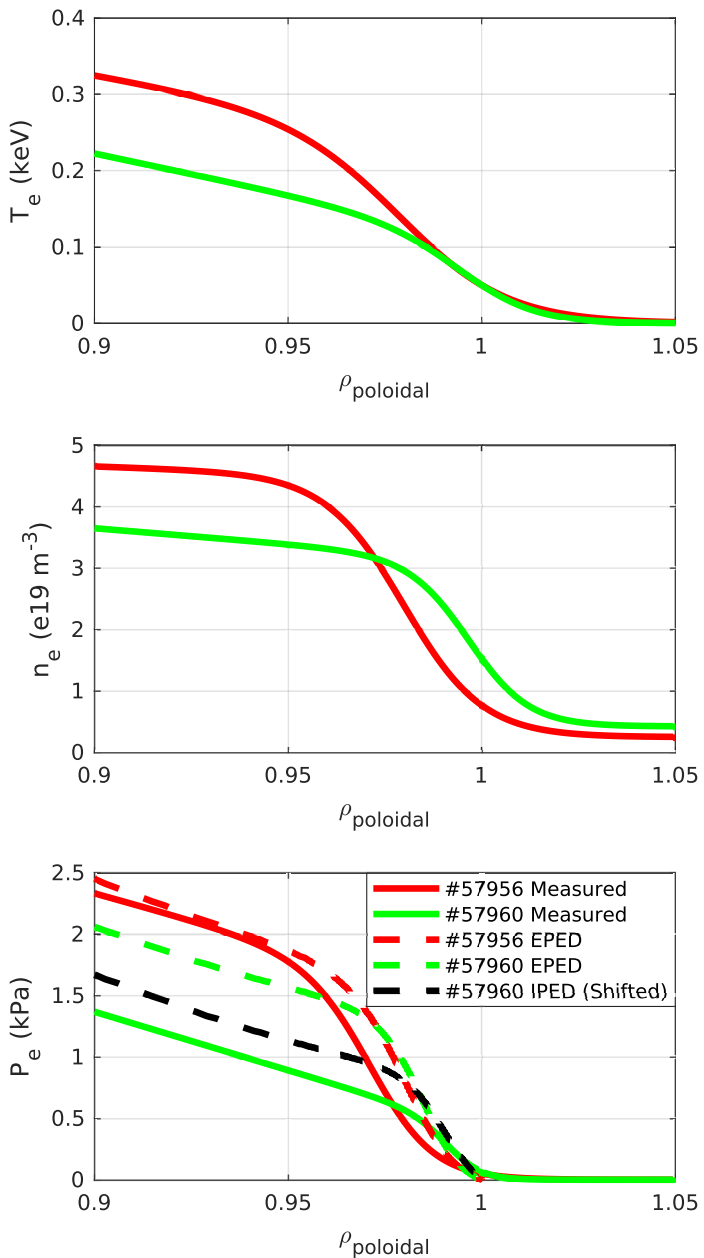

Figure 13. Pedestal profiles for discharges \#57956 (red) and \#57960 (green). IPED result for discharge \#57960 with shifted pedestal position simulations presented for pedestal $\mathrm{P}_{e}$ profiles.

\section{Conclusions}

Type I ELM-y beam heated scenarios were established with a high and low triangularity plasma on TCV. A series of discharges with varying rates of deuterium fuelling and/or nitrogen seeding were carried out to investigate the influence of gas injection on the pedestal parameters. Low nitrogen seeding lead to an increased pedestal top $\mathrm{P}_{e}$ but all other gas injection experiments resulted in a decrease. The low triangularity configuration was more resilient to high fuelling rates whereas the high triangularity discharges suffered a decrease of up to $80 \%$ in pedestal height. There was no clear trend between pedestal top $\mathrm{P}_{e}$ and total stored plasma energy, which was partially attributed to a lack of stiffness of the bulk plasma pressure profiles.

A PB stability analysis indicated that the discharges presented are on or near the ideal MHD stability limit validating the application of the EPED1 
model. However, the discharges did not follow the EPED1 relation $w=D \sqrt{\beta_{\theta}^{P e d}}$ with $D=0.076$. A $\sqrt{\beta_{\theta}^{P e d}}$ dependence has previously been shown on DIII-D and AUG and was therefore employed and a discharge specific constant $D$ was inferred from measurements. A wide range was found implying the profile gradient, set by transport, is having a strong effect in the discharges presented. EPED1 simulations were conducted using individual values of D, inferred from each discharge, and compared with simulations using the EPED1 constant value of 0.076. The EPED simulations with an inferred value of $D$ produced good agreement with experimental measurements, whilst poor agreement and minimal change in pedestal width and pedestal top $\mathrm{P}_{e}$ were observed with a constant value of 0.076 . This result suggests that these discharges are close to the ideal MHD stability limit and accurate values for $D$, a proxy for transport, are required.

Simulations using an experimentally inferred value of $D$ predicted pedestal top $\mathrm{P}_{e}$ values in good agreement for the high triangularity discharges but overestimated for the low triangularity. This was attributed to changes in pedestal position that are not captured with the EPED1 model. Modelling with IPED showed a $3 \%$ shift in pedestal position can result in a reduction of $50 \%$ in pedestal top $\mathrm{P}_{e}$ and produced better agreement with measurements at two extremes of the gas scan. This study has shown that the EPED model requires a scenario specific $D$ and accounting for the offset in temperature and density profiles to agree with the measured profiles.

\section{Acknowledgments}

This work has been carried out within the framework of the EUROfusion Consortium and has received funding from the Euratom research and training programme 2014-2018 under grant agreement No 633053. The views and opinions expressed herein do not necessarily reflect those of the European Commission.

[1] Wolfrum, E., et al., Nuclear Materials and Energy, 12, 18 (2017)

[2] Maggi, C., et al., Nuclear Fusion, 11, 113031 (2015)

[3] Maingi, R., et al., Physical Review Letters, 103, 075001 (2009)

[4] Kinsey, J. E., et al., Nuclear Fusion, 51.83001 (2011)

[5] Potzel, S., et al., Journal of Nuclear Materials, 463, 541 (2015)

[6] Reimold, F., et al., Nuclear Materials and Energy, 12, 193 (2017)

[7] Dunne, M. G., et al., Plasma Physics and Control Fusion, 59, 014017, (2017).

[8] Frassinetti, L., et al., Plasma Physics and Controlled Fusion, 59, 014014, (2017).

[9] Stefanikova, E., et al.,Nuclear Fusion, 58, 056010 (2018)

[10] Giroud, C. et al., Plasma Physics and Control Fusion, 57, $035004(2015)$
[11] Giroud, C. et al., Nuclear Fusion, 11, 113025 (2013)

[12] Dunne, M. G., et al., 26th IAEA FEC, Kyoto, Japan, (2016)

[13] Beurskens, M. N. A., et al., Nuclear Fusion, 56, 056014 (2016)

[14] Reimold, F., et al., Nuclear Materials and Energy 12, 193$199(2017)$

[15] Karpushov, A. N., et al.; Fusion Engineering and Design, 123468472 , (2017)

[16] Snyder, P., et al, Plasma Phys. Control Fusion, 45, 1671 (2003)

[17] Snyder, P. B., et al., Physics of Plasmas, 16, 056118, (2009).

[18] Frassinetti, L., et al., Nuclear Fusion, 57, 061012 (2017)

[19] Dunne, M. G., et al., Plasma Physics and Control Fusion, 59, 025010, (2017)

[20] Groebner, R. J., et al., Nuclear Fusion 41, 121789 (2001)

[21] Pitcher, C. S., and Stangeby, P. C., Plasma Physics and Controlled Fusion 39 779930, (1997)

[22] Stangeby, P. C. , The Plasma Boundary of Magnetic Fusion Devices, Bristol: Institute of Physics Publishing, (2000)

[23] Komm, M., et al. Contribution to the multi-machine pedestal scaling from the COMPASS tokamak, Nuclear Fusion 57.5, 056041, (2017)

[24] Merle, A., et al., Plasma Physics and Controlled Fusion, 59 104001, (2017)

[25] Ltjens, H., et al., Computer physics communications, 97.3 219-260, (1996)

[26] Sauter, O., et al., Physics of Plasmas, 62834 (1999)

[27] Medvedev., S. Y., et al., 20th EPS Conf. on Controlled Fusion and Plasma Physics, Lisbon, Proc. Contrib. Papers, Vol. 17C, p. 1279 Part IV, (1993)

[28] Degtyarev, L., et al. Computer Physics Communications, 103.1 10-27, (1997) 